\title{
Topological Quantum Computing with Read-Rezayi States
}

\author{
L. Hormozi, ${ }^{1}$ N. E. Bonesteel, ${ }^{2}$ and S. H. Simon ${ }^{3}$ \\ ${ }^{1}$ Joint Quantum Institute, National Institute of Standards and Technology, and University of Maryland, \\ Gaithersburg, Maryland 20899, USA \\ ${ }^{2}$ Department of Physics and NHMFL, Florida State University, Tallahassee, Florida 32310, USA \\ ${ }^{3}$ Rudolf Peierls Centre for Theoretical Physics, Oxford University, 1 Keble Road, Oxford OX1 3NP, United Kingdom
}

(Received 23 March 2009; published 13 October 2009)

\begin{abstract}
Read-Rezayi fractional quantum Hall states are among the prime candidates for realizing non-Abelian anyons which, in principle, can be used for topological quantum computation. We present a prescription for efficiently finding braids which can be used to carry out a universal set of quantum gates on encoded qubits based on anyons of the Read-Rezayi states with $k>2, k \neq 4$. This work extends previous results which only applied to the case $k=3$ (Fibonacci) and clarifies why, in that case, gate constructions are simpler than for a generic Read-Rezayi state.
\end{abstract}

DOI: 10.1103/PhysRevLett.103.160501

PACS numbers: 03.67.Lx, 03.67.Pp, 73.43.-f

Non-Abelian anyons [1]-quasiparticle excitations obeying so-called non-Abelian statistics-are conjectured to emerge in certain two-dimensional quantum systems. In these systems, when well-separated anyons are present, there is a ground state degeneracy that grows exponentially with the number of anyons. Furthermore, if these anyons remain well separated, different states in the ground state manifold cannot be distinguished by local measurements-thus rendering this space immune from decoherence due to any local perturbations.

When non-Abelian anyons are exchanged, the process is described by a multidimensional unitary operation (instead of a single phase) acting on the degenerate space. Certain unitary operations can then be carried out by dragging anyons around one another, "braiding" their worldlines in $2+1$ dimensional space-time. As long as the anyons are kept sufficiently far apart during this process, the resulting unitary operations will be identical for any two topologically equivalent braids.

Recent interest in non-Abelian anyons has focused on the possibility of using them for topological quantum computation- a form of quantum computation which exploits the topological robustness of braiding and the protection of the degenerate Hilbert space against decoherence to process and store quantum information in an intrinsically fault-tolerant way [2-5]. This Letter is concerned with the problem of finding specific braiding patterns which can be used to carry out universal quantum computation for a class of non-Abelian anyons described by $\mathfrak{S} \mathfrak{H}(2)_{k}$ Chern-Simons-Witten theories.

The theory of $\mathfrak{g} \mathfrak{l}(2)_{k}$ anyons provides the mathematical description [6] (up to global Abelian phases not relevant here) of the braiding properties of quasiparticle excitations in the Read-Rezayi [7] sequence of fractional quantum Hall (FQH) states. Here, the parameter $k$ (the "level") is a positive integer characterizing the state. For example, the $k=2$ state is the Moore-Read state [1], believed to describe the FQH plateau observed at filling fraction $\nu=5 / 2$
[4], and the $k=3$ state may describe the $\mathrm{FQH}$ plateau observed at $\nu=12 / 5$ [7]. Bosonic Read-Rezayi states may also be realizable in rotating Bose gases [8], and model lattice Hamiltonians have been constructed [9] for which the low-energy quasiparticle excitations can be described by any consistent achiral theory of non-Abelian anyons, including (doubled) $\mathfrak{S} \mathfrak{H}(2)_{k}$, suggesting the possibility of realizing $\mathfrak{S} \mathfrak{H}(2)_{k}$ anyons in exotic spin liquids.

It has been shown that $\mathfrak{g} \mathfrak{u}(2)_{k}$ anyons with $k=3$ or $k>$ 4 can be used to carry out universal quantum computation just by braiding anyons [3]. In previous work [10,11], different prescriptions have been given for explicitly constructing braids to carry out universal quantum computation using $\mathfrak{S} \mathfrak{u}(2)_{3}$ anyons-anyons which, for our purposes, are essentially equivalent to the so-called Fibonacci anyons $[4,10]$. These prescriptions all exploited unique properties of Fibonacci anyons and so could not be straightforwardly generalized to other anyon models. In the present work, we introduce a class of constructions which can be applied to all $\mathfrak{G} \mathfrak{i t}(2)_{k}$ anyons with $k \geq 3, k \neq 4$.

$\mathfrak{S} \mathfrak{u}(2)_{k}$ anyons carry a quantum number resembling ordinary spin referred to here as (topological) "charge" $[4,12]$. For the level $k$ theory, the allowed values of this charge include all integers and half integers between 0 and $k / 2$. Similar to ordinary spin, there are rules for combining topological charge which specify the possible total charge of objects formed when two or more anyons are combined. For $\mathfrak{S H}(2)_{k}$ anyons, the fusion rule is a truncated version of the usual triangle rule for adding angular momenta,

$$
\begin{aligned}
s_{1} \otimes s_{2}= & \left|s_{1}-s_{2}\right| \oplus\left(\left|s_{1}-s_{2}\right|+1\right) \oplus \cdots \oplus \min \left[s_{1}+s_{2}, k\right. \\
& \left.-\left(s_{1}+s_{2}\right)\right] .
\end{aligned}
$$

From this fusion rule, it can be shown that, asymptotically, the dimensionality of the Hilbert space of $N$ identical anyons grows as $d_{k}^{N}$, where $d_{k}$ is known as the quantum dimension of the particles $[4,12]$. For charge $1 / 2$ anyons 
$d_{k}=\lfloor 2\rfloor_{q}$, where the $q$ integer $\lfloor m\rfloor_{q}$ is defined as $\lfloor m\rfloor_{q}=$ $\left(q^{m / 2}-q^{-m / 2}\right) /\left(q^{1 / 2}-q^{-1 / 2}\right)$ and $q=e^{i 2 \pi /(k+2)}$.

For all finite $k \geq 2$, the quantum dimension is an irrational number between 1 and 2. It follows that the Hilbert space of $N$ charge $1 / 2, \mathfrak{S H}(2)_{k}$ anyons cannot be decomposed into a tensor product of smaller subsystems. To carry out quantum computation within the standard "qubit plus quantum gate" framework, one must therefore encode qubits using several anyons. Here we encode qubits in the two-dimensional Hilbert space of four charge 1/2 anyons with total charge 0 [5]. Referring to Fig. 1, we choose the logical $|0\rangle$ and $|1\rangle$ for this qubit to be states for which the total charge of the two bottommost (or, equivalently, the two topmost) anyons is 0 and 1 , respectively. Note that the fusion rule (1) indicates that, when the constraint that the total charge be 0 is relaxed, the Hilbert space of four charge $1 / 2$ anyons is five dimensional for $k=3$ and six dimensional for $k>3$. The states that do not correspond to qubit states, i.e., states with total charge 1 or 2 , are then noncomputational states. The possibility of transitions from the qubit space to this noncomputational space, i.e., leakage errors, poses a major problem in constructing quantum gates.

To carry out universal quantum computation, it must be possible to perform arbitrary single-qubit gates as well as at least one entangling two-qubit gate. It has been shown that for $\mathfrak{S} \mathfrak{t}(2)_{k}$ anyons, when $k \geq 3, k \neq 4,8$, any desired single-qubit gate can be approximated, to any desired accuracy, by weaving a charge $1 / 2$ anyon around two others within a qubit $[3,13]$. In practice, braids which approximate a given single-qubit gate can be found by carrying out a search over braids up to a given length and choosing those which produce unitary operations closest to the desired target gate. As an example, Fig. 1 shows a braid which is the result of a bidirectional search [14] and which approximates a Hadamard gate using $\mathfrak{S} \mathfrak{H}(2)_{5}$ anyons to an

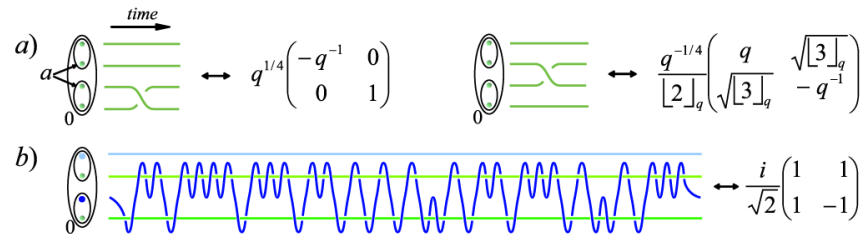

FIG. 1 (color online). (a) Encoded qubits and elementary braid matrices for $\mathfrak{s} \mathfrak{H}(2)_{k}$ anyons and (b) a sample single-qubit gate. Here, and in subsequent figures, groups of anyons in charge eigenstates are enclosed in ovals labeled by the charge. Qubits are encoded in the two-dimensional Hilbert space of four charge $1 / 2$ anyons with total charge 0 . The matrices shown, in the basis labeled by $a=\{0,1\}$, correspond to the elementary braid operations, also shown. Within the encoded qubit space, braiding the top two anyons produces the same unitary operation as braiding the bottom two. The braid depicted in (b) approximates a Hadamard gate for $\mathfrak{s} \mathfrak{H}(2)_{5}$ anyons with an accuracy of $\sim 2.2 \times$ $10^{-6}$ (measured using operator norm; see [10]). accuracy of 1 part in $10^{6}$. Given the ability to perform a search this deep, further accuracy can always be systematically achieved by applying the Solovay-Kitaev algorithm [10]. Note that for single-qubit gates, there is no danger of leakage errors because braiding within a qubit cannot change its total topological charge.

When searching for braids, we are performing a discrete search over a continuous space. The dimensionality of the space being searched, $D$, is the main factor which determines the efficacy of these searches. If the number of distinct braids one can search is $N_{b}$, the typical error for approximating a given target gate will be $\sim N_{b}^{-1 / D}$. Thus, for fixed $N_{b}$, increasing $D$ greatly reduces the accuracy of the braids one can obtain. For single-qubit gates, the search space is $S U(2)$ with $D=3$, which is sufficiently small to allow us to find highly accurate braids, as demonstrated by the above example.

Two-qubit gates are significantly harder to construct, both due to the possibility of leakage errors and the fact that for eight anyons the search space is $S U(13)$ for $k=3$ and $S U$ (14) for $k>3$ (with $D=168$ and 195, respectively). The key idea for efficiently finding braids for two-qubit gates, introduced in [10] and common to more recent approaches [11], is to reduce the two-qubit gate problem to one or more effective "single-qubit" problems, i.e., problems in which one is searching over braids which involve only three objects at time (where an object can be either a single anyon or a collection of anyons braided as a single entity) and for which the search space is $S U(2)$.

In addition to reducing all searches to $S U(2)$, previous work has focused on the case $k=3$. When $k=3$, for charge 1 anyons, the only nontrivial fusion rule is $1 \otimes 1=0 \oplus 1$ - the fusion rule of the Fibonacci anyons [4]. All previous gate constructions have exploited the unique feature of Fibonacci anyons that any collection of them will either have topological charge 0 and therefore be "neutral" (i.e., not induce non-Abelian transitions if braided as a single cohesive object) or topological charge 1 and thus behave as a single Fibonacci anyon.

We illustrate the usefulness of this feature with a simple two-qubit gate construction [15] (see also [11]). Figure 2(a) shows a two-qubit braid in which a pair of anyons from the control qubit (the control pair) is woven around pairs of anyons in the target qubit before returning to its original position. When the control qubit is in the state $|0\rangle$, the control pair has charge 0 and the result of this operation will be trivial (i.e., the identity). Similarly, when the target qubit is in the state $|0\rangle$, the control pair is woven around objects with total charge 0 and the result will again be the identity (regardless of the state of the control qubit). Thus, by construction, this weave acts as the identity on the twoqubit states $|0\rangle|0\rangle,|0\rangle|1\rangle$, and $|1\rangle|0\rangle$, with the only nontrivial case being $|1\rangle|1\rangle$ (here the first qubit is the control qubit). To construct an entangling two-qubit gate, it is then necessary to find a particular weave of the form shown in 


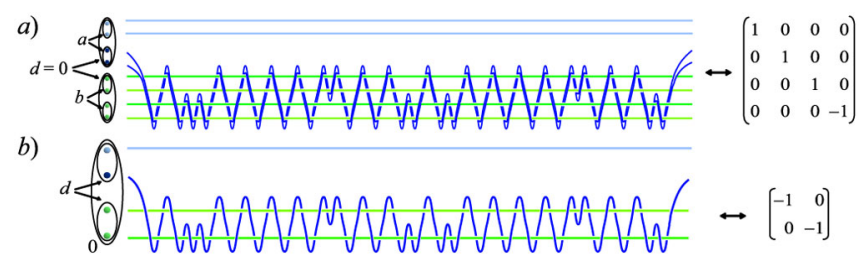

FIG. 2 (color online). "Effective qubit" gate construction for $\mathfrak{S} 1 \mathrm{t}(2)_{3}$ anyons. Part (a) shows a braid in which a pair of anyons from the control qubit (blue) weaves around pairs of anyons in the target qubit (green). When either qubit is in the state $|0\rangle$, this braid produces the identity operation. When both control and target qubits are in the state $|1\rangle$, the braid consists of weaving a charge 1 anyon around two other charge 1 anyons, as shown in (b). For $\mathfrak{s l t}(2)_{3}$ anyons, the braiding properties of charge $1 / 2$ anyons and charge 1 anyons are the same (up to irrelevant Abelian phases), and the problem is reduced to that of finding a particular single-qubit gate by carrying out a search in $S U(2)$. The braid shown in (b) approximates a negative identity matrix, in the basis labeled by $d=\{0,1\}$, for $\mathfrak{S H}(2)_{3}$ anyons with an accuracy of $\sim 1.6 \times 10^{-5}$. As a consequence, the full braid shown in (a) approximates a controlled- $Z$ gate, shown in the basis labeled by $a b=\{00,01,10,11\}$, with an accuracy of $\sim 1.4 \times 10^{-5}$.

Fig. 2(a) which returns the state $|1\rangle|1\rangle$ to itself while acquiring a nontrivial phase with respect to the state $|1\rangle|0\rangle$, thus producing a controlled rotation of the target qubit.

Finding such a weave is straightforward for Fibonacci anyons $(k=3)$. In this case, the fusion rule implies the Hilbert space of four charge 1 objects with total charge 0 is two dimensional and the problem reduces to that of searching for a particular single-qubit operation acting on the "effective qubit" shown in Fig. 2(b). The states of this "qubit" are determined by the label $d$ which can be either 0 or 1 . To ensure there are no transitions between the encoded qubit states $(d=0)$ and the noncomputational states $(d=1)$, the resulting unitary operation must be diagonal in $d$. As an example, Fig. 2(b) shows a braid which approximates a negative identity matrix. If one follows this braiding pattern by weaving the control pair around pairs of anyons in the target qubit [as shown in Fig. 2(a)], the $|1\rangle|1\rangle$ state acquires a phase of -1 and the resulting two-qubit gate is a controlled- $Z$ gate which is equivalent to a CNOT up to single-qubit rotations.

We now turn to the case $k>3$. In this case, the fusion rule for combining two charge 1 objects is $1 \otimes 1=0 \oplus 1 \oplus$ 2 . This implies that $d$, the overall charge of the original qubits shown in Fig. 2, can now take three different values. The unitary operations corresponding to braids of the form shown in Fig. 2(a) are then elements of $S U(3)$, not $S U(2)$. While it is in principle possible to carry out a search in $S U(3)$, because the dimensionality of the search space is 8 , rather than 3 ,it is significantly less efficient than searching $S U(2)$.

In general, we find that for $k>3$ it is impossible to construct a leakage free two-qubit entangling gate by performing a single braid in which three objects are braided and the search space is $S U(2)$ [16]. However, it is possible to construct such a gate by breaking the construction into three steps, as illustrated in Fig. 3. In each of these steps, a pair of anyons from the control qubit (again, the control pair) is woven around two anyons in the target qubit.

Before describing the details of this construction, we establish the key fact that finding braids for each step only requires a search in $S U(2)$. As before, we need only consider the case when the control pair has charge $1(a=1)$. Each step then involves weaving a charge 1 object around two charge $1 / 2$ anyons. According to the fusion rule (1), the Hilbert space of these three objects decouples into two one-dimensional sectors (with total charge 0 and 2) and a single two-dimensional sector (with total charge 1). The action on the one-dimensional sectors is determined entirely by the winding number of the braid-in particular, if we fix the winding number to be 0 the action is trivially the identity [10]. The only nontrivial action is then on the twodimensional sector, for which the relevant search space is $S U(2)$. For $\mathfrak{G} \mathfrak{H}(2)_{k}$ anyons, it is straightforward to deter-

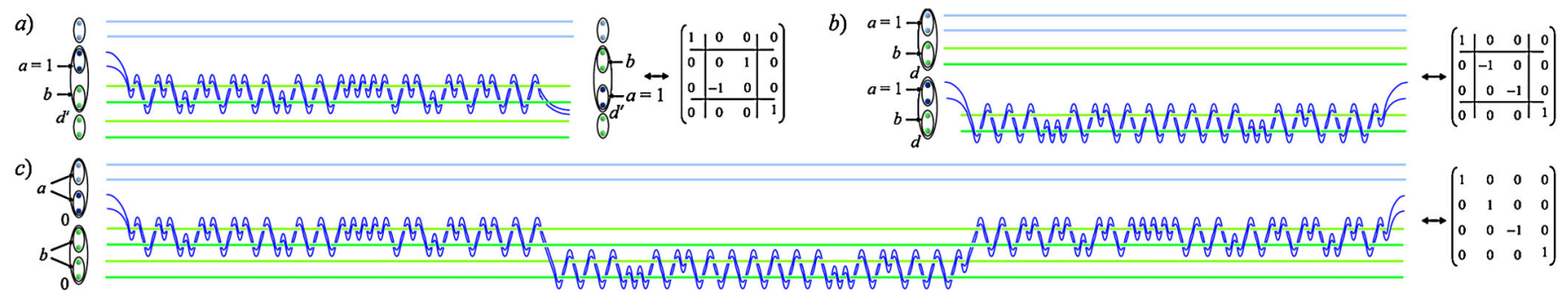

FIG. 3 (color online). Controlled-phase gate construction for $\mathfrak{s} \mathfrak{u}(2)_{5}$ anyons. Part (a) shows a swap braid which exchanges the control pair (blue) with the topmost pair of anyons in the target qubit (green). This braid approximates the unitary operation shown in the figure with an accuracy of $\sim 5.1 \times 10^{-6}$. Note that the matrix shown is expressed using inequivalent left and right basis states labeled $b d^{\prime}=\{10,01,11,12\}$ at both the start and end of the braid. Part (b) shows a braid which acts on the intermediate state produced by the swap braid and gives the state with $a=1 b=0$ a phase of -1 . The corresponding unitary operation shown in the figure in the basis labeled by $b d=\{10,01,11,12\}$ is approximated with an accuracy of $\sim 7.0 \times 10^{-6}$. (c) Full controlled- $(-Z)$ gate, in the basis labeled by $a b=\{00,01,10,11\}$, with an accuracy of $\sim 7.4 \times 10^{-7}$. 
mine the relevant braid matrices [12,15], and we find that for all $k \geq 3, k \neq 4$ these matrices generate dense covers of $S U(2)$.

Now we turn to the actual construction. The first step consists of a braid which effectively "swaps" the control pair with a pair of anyons in the target qubit. Figure 3(a) shows this step for $\mathfrak{g} \mathfrak{H}(2)_{5}$ anyons. Referring to this figure, the control pair starts from the bottom position in the control qubit, weaves around the two topmost anyons in the target qubit, and swaps positions with them in the end. The specific braid shown is the result of a search for a braid which generates a unitary operation approximating the matrix shown in the figure. (In this matrix-and the one shown for step 2-the upper left and lower right elements correspond to the one-dimensional sectors with total charge 0 and 2 , and the middle $2 \times 2$ block acts on the two-dimensional sector with total charge 1 , described above.) This operation is designed to effectively swap the control pair with the topmost pair of anyons in the target qubit (labeled $b$ ), and when $a=b=1$, it does so without disturbing the quantum numbers of the system. This means that if the initial state of the qubit is $|1\rangle|1\rangle$, the final state will be the same but with the control pair now swapped into the target.

The net effect of this swap operation is to take the system to the intermediate state shown as the starting state in Fig. 3(b). In this intermediate state, all of the information of the initial state of the two qubits is encoded in the bottom four anyons. In particular (assuming $a=1$ ), the total charge of these anyons, labeled $d$ in the figure, must be 0 if $b=1$ (since in this case the swap operation is essentially the identity) and 1 if $b=0$ (due to the fusion rules).

To perform an entangling two-qubit gate, we need only induce a phase shift between the $b=0$ and $b=1(d=0)$ states when $a=1$. This is done in the second step of our construction, in which the control pair is woven around the bottom two anyons and returned to its starting position [see Fig. 3(b)]. The weave shown is the result of a search which produces a unitary operation that is diagonal in $b$ and gives the state $a=1, b=0$ a nontrivial phase ( -1 for the braid shown).

In the third and final step, the control pair is returned to its original position in the control qubit by applying the inverse of the swap braid. Putting all three steps together, the resulting full braid is shown in Fig. 3(c). If the control qubit is in the state $|0\rangle$, the control pair has charge 0 and the effect of this braid is simply the identity operation. If the control qubit is in the state $|1\rangle$, this braid first swaps the control pair into the target, then, if the target qubit was initially in the state $|0\rangle$, gives the resulting intermediate state a phase $e^{i \phi}$, and finally returns the control pair to the control qubit. The full braid then approximates a controlled-phase gate for which, if the control qubit is in the state $|1\rangle$, the target qubit is rotated about the $\hat{z}$ axis by the angle $\phi$. When $\phi=\pi$, as is the case for the braid shown in the figure, this gate is a controlled- $(-Z)$ gate which is equivalent to a CNOT up to single-qubit rotations.

We conclude by pointing out that for certain values of $k$, it is possible to carry out step 2 of our construction with a finite braid in such a way that the phase difference between the states $a=1, b=0$ and $a=1, b=1(d=0)$ is exactly -1 . Specifically, for $k=8 n-2$ this can be done by weaving the control pair completely around the two bottom anyons in the target qubit $n$ times.

We gratefully acknowledge Wayne Witzel for developing some of the codes used to find the braids shown in this Letter. We also thank the Aspen Center for Physics for its hospitality during the completion of part of this work and ICAM for providing travel support (L.H.). We acknowledge support from NIST/NRC (L. H.) and U.S. DOE Grant No. DE-FG02-97ER45639 (N. E. B.).

[1] G. Moore and N. Read, Nucl. Phys. B360, 362 (1991).

[2] A. Yu. Kitaev, Ann. Phys. (N.Y.) 303, 2 (2003); 321, 2 (2006).

[3] M. Freedman et al., Commun. Math. Phys. 227, 605 (2002); 228, 177 (2002).

[4] For a review, see C. Nayak et al., Rev. Mod. Phys. 80, 1083 (2008), and references therein.

[5] M. Freedman et al., Bull. Am. Math. Soc. 40, 31 (2002); J. Preskill, "Lecture Notes for Physics 219: Quantum Computation-Part III: Topological Quantum Computation," available at http://www.theory.caltech.edu/ $\sim$ preskill/ph219/topological.pdf.

[6] J. K. Slingerland and F. A. Bais, Nucl. Phys. B612, 229 (2001); E. Ardonne and K. Schoutens, Ann. Phys. (N.Y.) 322, 201 (2007).

[7] N. Read and E. Rezayi, Phys. Rev. B 59, 8084 (1999); E. H. Rezayi and N. Read, Phys. Rev. B 79, 075306 (2009).

[8] N. R. Cooper et al., Phys. Rev. Lett. 87, 120405 (2001).

[9] M. Levin and X.-G. Wen, Phys. Rev. B 71, 045110 (2005); P. Fendley and E. Fradkin, ibid. 72, 024412 (2005).

[10] N. E. Bonesteel et al., Phys. Rev. Lett. 95, 140503 (2005); L. Hormozi et al., Phys. Rev. B 75, 165310 (2007).

[11] H. Xu and X. Wan, Phys. Rev. A 78, 042325 (2008); 80, 012306 (2009).

[12] See, for example, J. Fuchs, Affine Lie Algebras and Quantum Groups (Cambridge University Press, Cambridge, 1992).

[13] S. H. Simon et al., Phys. Rev. Lett. 96, 070503 (2006).

[14] See, for example, G. Zikos et al., Int. J. Mod. Phys. B 23, 2727 (2009).

[15] L. Hormozi, Ph.D. thesis, Florida State University, 2007.

[16] One can consider constructing two-qubit gates by weaving the control pair around two anyons in the target qubit or around a pair and a single anyon; however, the former cannot lead to an entangling two-qubit gate when $k>3$ [10], while the latter requires a search in $S U(2) \oplus S U(2)$ with a six-dimensional search space. 